\title{
Long-read genome assemblies uncover extensive loss of micro-syntenty and conservation of macro-synteny among three woodland Eucalyptus species.
}

\author{
Scott Ferguson ${ }^{1}$, Ashley Jones ${ }^{1}$, Kevin Murray ${ }^{1}$, Benjamin Schwessinger $^{1}$, and Justin \\ Borevitz ${ }^{1}$
}

\author{
${ }^{1}$ Australian National University
}

February 7, 2022

\begin{abstract}
Synteny, the ordering of sequences within homologous chromosomes, must be maintained within the genomes of sexually reproducing species for the sharing of alleles and production of viable, reproducing offspring. However, when the genomes of closely related species are compared, a loss of synteny can be observed. Synteny loss may be occurring due to transposonmediated recombination errors during meiosis, unequal homologous recombination, resulting in the formation of chromosomal rearrangements. To examine patterns of synteny amongst three closely related, interbreeding, and wild Eucalyptus species, we assembled their genomes using long-read DNA sequencing and de novo assembly. We identify syntenic and rearranged regions between these genomes and estimate that $\sim 48 \%$ of our genomes remain syntenic while $\sim 36 \%$ is rearranged. We observed that rearrangements highly fragment micro-synteny, however genome-wide, macro-synteny appears to be maintained. Our results suggest that synteny between our species is primarily lost through small-scale rearrangements, not through sequence loss, gain, or sequence divergence. Further examination of identified rearrangements suggests that rearrangements may be altering the phenotypes of Eucalyptus species. Our study also questions the use of single reference genomes in genomic studies, reliance on a single reference genome when seeking genetic variation leads to reference bias, especially given the scale at which we show potentially adaptive loci have highly diverged, deleted, duplicated and/or rearranged. This study provides an unbiased framework to look at potential speciation and adaptive loci among a rapidly radiating foundation species of woodland trees that are free from selective breeding seen in most crop species.
\end{abstract}

\section{Hosted file}

Main Document.docx available at https://authorea.com/users/459343/articles/555631-long-readgenome-assemblies-uncover-extensive-loss-of-micro-syntenty-and-conservation-of-macrosynteny-among-three-woodland-eucalyptus-species 